Original Article

\title{
GC-MS ANALYSIS OF INVASIVE AQUATIC WEED, PISTIA STRATIOTES L. AND EICHHORNIA CRASSIPES (MART.) SOLMS
}

\author{
TULIKA TYAGI ${ }^{*}$, MALA AGARWAL ${ }^{\mathrm{a}}$ \\ ${ }^{*}$ Faculty of Biotechnology, B. B. D. Government P. G. College, Chimanpura, Jaipur, University of Rajasthan, Jaipur Rajasthan State India \\ Email: tulikatyagi_062@yahoo.co.in
}

Received: 02 Jan 2017, Revised and Accepted: 31 Apr 2017

\section{ABSTRACT}

Objective: To investigate the bioactive components of an invasive aquatic weed, Pistia stratiotes L. and Eichhornia crassipes (Mart.) Solms vegetative parts by using Gas Chromatography-Mass Spectrometer (GC-MS).

Methods: The chemical compositions of the ethanol extract of whole plant Pistia stratiotes L. and Eichhornia crassipes (Mart.) Solms was investigated using Agilent Technologies GC-MS (GC-7890A, MS 5975C).

Results: The results of GC-MS analysis of the ethanolic extract revealed the existence of 28 phytochemical compounds in Pistia stratiotes L. nHexadecanoic acid,-11-Hexadecenoic acid, ethyl ester, Hexadecanoic acid, ethyl ester, Octadecanoic acid, ethyl ester, 2-Cyclopenten-1-one, 5hydroxy-2,3-dimethyl, L-Glutamine, 2-Pentadecanone, 6,10,14-trimethyl, Linolelaidic acid, methyl ester, 9,12,15-Octadecatrienoic acid, methyl ester,(Z,Z,Z), Nonadecane, 12,15-Octadecadiynoic acid, methyl ester, Hexadecanoic acid, 2-hydroxy-1-(hydroxymethyl)ethyl ester, Diisooctyl phthalate, Docosanoic acid, ethyl ester, Stigmasterol, Bis(2-ethylhexyl) phthalate, 1-Monolinoleoylglycerol trimethylsilyl ether, Ethyl iso-allocholate are the major compound.

The ethanolic extract of Eichhornia crassipes (Mart.) Solms contains 43 phytochemical compounds of high and low molecular weight nHexadecanoic acid, E-11-Hexadecenoic acid, ethyl ester, Palmitic acid, Phytol, 9,12,15-Octadecatrienal, 9,12-Octadecadienoic acid, ethyl ester, Linolenic acid, ethyl ester, Stearic acid, ethyl ester, Hexadecanoic acid, 2-hydroxy-1-(hydroxymethyl)ethyl ester, $\alpha$-Glyceryl linolenate, 1Monolinoleoylglycerol trimethylsilyl ether, Linoleic acid, 2,3-bis-(0-TMS)-propyl ester, Stigmasterol, Linolelaidic acid, methyl ester, 9,12,15Octadecatrienoic acid, ethyl ester, (Z,Z,Z), Ethyl iso-allocholate, Cholesta-22,24-dien-5-ol, 4,4-dimethyl are the major compounds.

These results indicates Pistia stratiotes L. and Eichhornia crassipes (Mart.) Solms possess potent antioxidant, anti-inflammatory, anticancer, antitumour, antiarthritic, cancer preventive, antibacterial effects so can be recommended as a plant of phytopharmaceutical importance.

Conclusion: The ethanol extract of Pistia stratiotes L. and Eichhornia crassipes (Mart.) Solms proves as a potential source of bioactive compounds of pharmacological importance.

Keywords: Antioxidant, Anti-inflammatory, Anticancer, Antitumour, Antiarthritic

(C) 2017 The Authors. Published by Innovare Academic Sciences Pvt Ltd. This is an open access article under the CC BY license (http://creativecommons.org/licenses/by/4.0/) DOI: http://dx.doi.org/10.22159/ijcpr.2017v9i3.19970

\section{INTRODUCTION}

Plants are valuable for modern folklore medicine as they are sources of direct therapeutic agents. Herbal plants produce and contain a variety of chemical substances, of these substances certain isolated compounds serve as models for new synthetic compounds and can be used as taxonomic markers for the discovery of new compounds. However, only a fraction of the world's plants has been studied. Over the years evaluation of crude drugs has undergone many changes.

Due to advancement in the chemical knowledge of crude drugs, evaluation methods include estimating active constituents present in the crude drug, in addition to its morphological and microscopic analysis. Quality control standardisations of the various medicinal plants used in traditional medicine is now becoming more important today in view of the commercialization of formulations based on these plants [1].

Pistia stratiotes, commonly known as water cabbage or water lettuce, belongs to the family Araceae, is an edible, aquatic, floating ornamental plant with widely distributed across tropical and subtropical areas around the world. P. stratiotes is widely distributed and is being loathed in Asia and Africa. This plant and its extracts potentially have medicinal effects. In various parts of the world, it is also used as anodyne for eyewash. The leaves are used in eczema, leprosy, ulcers and piles [2]. The plant is bitter, pungent flavor, having cooling, laxative property. It is used in 'Tridosha' fever and diseases of the blood. Leaf infusions have been mentioned in the folklore to be used for dropsy, bladder complaints, kidney afflictions, hematuria, dysentery and anemia [3].

The freshwater aquatic plant $E$. crassipes, commonly known as water hyacinth is a member of the family Pontederiaceae. This fast growing, free-floating, perennial plant is indigenous to Brazil Amazon basin and Ecuador region. It was introduced as an ornamental species to adorn the water bodies. Water hyacinth is a source of many compounds with radical scavenging activity, such as vitamins, terpenoids, phenolic acids, lignin, stilbens, alcaloids, sterols, and other metabolites with high antioxidant activity [4]. Phytosterols are steroidal molecules that show a similar structure to cholesterol found in many vegetables such as water hyacinth. The most common phytosterol compounds is stigma sterol. Those compounds comprise $98 \%$ of all the vegetable sterols identified in plants [5].

The aim of this study was to analyze organic water lettuce and water hyacinth extracts through phytochemical screening and gas chromatography-mass spectrometry (GC-MS) to elucidate their chemical composition and to determine their potential applications.

\section{MATERIALS AND METHODS}

\section{Collection of plant material}

Leaves of Pistia stratiotes L. (Araceae) and Eichhornia crassipes (Mart.) solms, (Pontederiaceae), were collected from Kishor Sagar lake, Kota city, Rajasthan, India. It is situated between $25^{\circ} 11^{\prime} 0 " \mathrm{~N}$ 
latitude and $75^{\circ} 50^{\prime} 0^{\prime \prime} \mathrm{E}$ longitude. Kishor Sagar lake in Kota city is one of the major water bodies enhances the groundwater level around this area.

\section{Preparation of samples}

The collected plant materials were air-dried and ground into uniform powder. Dry powder of plant sample was extracted with ethanol using soxhlet apparatus for $6 \mathrm{~h}$. The extract was filtered, followed by concentrated using rotary evaporator. The concentrated extract was subjected to freeze drying in a lyophilizer till dry powder was obtained. Finally, the extracted powder was suspended with the ethonal at the concentration of $100 \mathrm{mg} / \mathrm{ml} \mathrm{(w/v)} \mathrm{followed}$ by filtration through Varian Bond Elute C18 solid phase extraction to remove impurities. $1 \mu \mathrm{l}$ of this solution was employed for GC-MS-MS analysis.

\section{Gas chromatography-mass spectroscopy analysis}

The GC-MS analysis was carried out using Agilent Technologies GCMS (GC-7890A, MS 5975C) with Fused silica $15 \mathrm{~m}$ x $0.2 \mathrm{~mm}$ ID x $1 \mu \mathrm{m}$ of the capillary column. The instrument was set to an initial temperature of $110{ }^{\circ} \mathrm{C}$, and maintained at this temperature for 2 $\mathrm{min}$. At the end of this period, the oven temperature was rose up to $280{ }^{\circ} \mathrm{C}$, at the rate of an increase of $5^{\circ} \mathrm{C} / \mathrm{min}$, and maintained for 9 min. Injection port temperature was ensured as $250{ }^{\circ} \mathrm{C}$ and Helium flow rate as $1 \mathrm{ml} / \mathrm{min}$. The ionisation voltage was $70 \mathrm{eV}$. The samples were injected in split mode as 10:1. Mass spectral scan range was set at 30-450 (m/z). Using computer searches on a NIST Ver.2.1 MS data library and comparing the spectrum obtained through GC-MS compounds present in the plant's sample were identified.

Interpretation on mass-spectrum GC-MS was conducted using the database of National Institute Standard and Technology (NIST) having more than 62,000 patterns. The spectrum of the unknown components was compared with the spectrum of known components stored in the NIST library. The name, molecular weight and structure of the components of the test materials were ascertained.

\section{RESULTS AND OBSERVATION}

Gas chromatogram and mass spectra of different plant parts of ethanol extract of Pistia stratiotes L. and Eichhornia crassipes (Mart.) solms are presented in fig. 1, 2, 3, 4 and 5 respectively. The detailed tabulation of GC-MS analysis of the extract is given in table 1, table 2, table 3 , table 4 and table 5 respectively. The activity of the compounds as reported in Dr. Duke's Phytochemical and Ethno botanical Databases which are screened during GC-MS analysis justify the traditional medicinal uses.

\section{Sample Ref No: 548/C-101/07-16 \\ Sample Name: $\$ 4$ \\ Abundance}

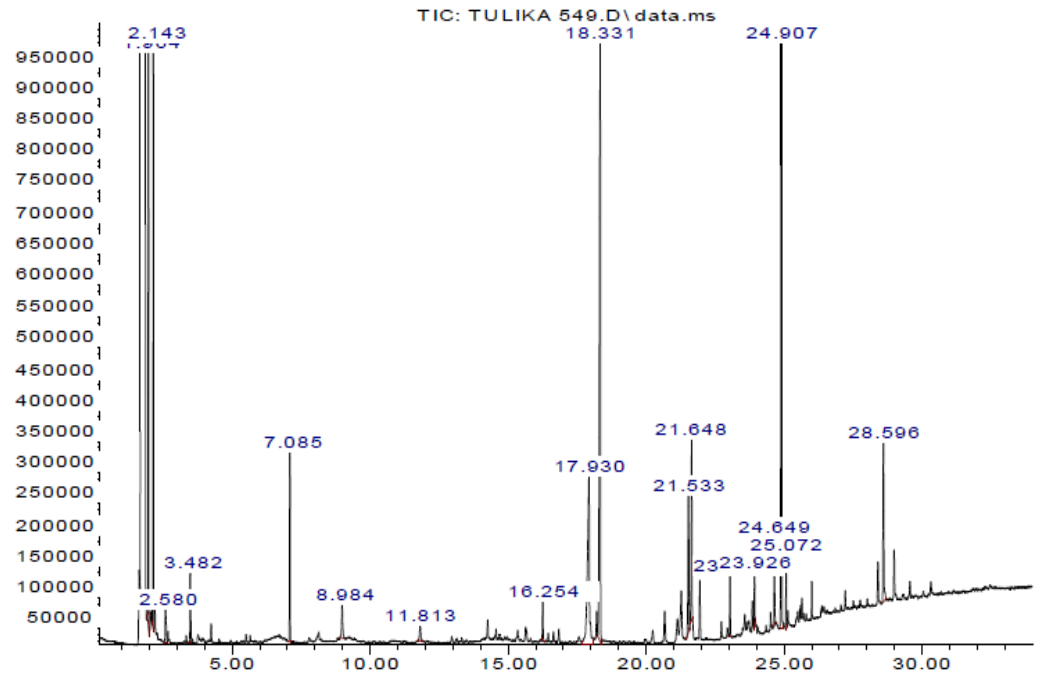

Fig. 1: GC-MS Spectra of ethanolic extract of Leaf of $P$. stratiotes

Table 1: Photo component present in the ethanolic extract of the Leaf of Pistia stratiotes by GC-MS

\begin{tabular}{|c|c|c|c|c|c|c|}
\hline RT & Name of Compound & MF & MW & Peak area \% & Compound nature & Activity \\
\hline 2.14 & Isobutyl alcohol & $\mathrm{C}_{4} \mathrm{H}_{10} \mathrm{O}$ & 74 & 5.42 & Alcohol & Biofuel \\
\hline 3.48 & $\begin{array}{l}\text { Formic acid,1-methylethyl } \\
\text { ester }\end{array}$ & $\mathrm{C}_{4} \mathrm{H}_{8} \mathrm{O}_{2}$ & 88 & 0.93 & Carboxylic acid & $\begin{array}{l}\text { Preservative, antibacterial agent, treatment } \\
\text { for warts. }\end{array}$ \\
\hline 7.08 & $\begin{array}{l}\text { Propane,1,1-diethoxy-2- } \\
\text { methyl }\end{array}$ & $\mathrm{C}_{8} \mathrm{H}_{18} \mathrm{O}_{2}$ & 146 & 2.05 & Ether & No activity reported. \\
\hline 11.81 & L-Glutamine & $\mathrm{C}_{5} \mathrm{H}_{10} \mathrm{~N}_{2} \mathrm{O}_{3}$ & 146 & 0.38 & Amino acid & Building block of Protein \\
\hline 17.93 & n-Hexadecanoic acid & $\mathrm{C}_{16} \mathrm{H}_{32} \mathrm{O}_{2}$ & 256 & 7.18 & $\begin{array}{l}\text { Palmitric acid } \\
\text { (saturated fatty } \\
\text { acid) }\end{array}$ & $\begin{array}{l}\text { Antioxidant, Hypocholesterolemic, } \\
\text { Nematicide, Pesticide, Lubricant, } \\
\text { Antiandrogenic, Hemolytic, 5-alpha } \\
\text { reductase inhibitor, antipsychotic }\end{array}$ \\
\hline 18.33 & $\begin{array}{l}\text { Hexadecanoic acid, ethyl } \\
\text { ester }\end{array}$ & $\mathrm{C}_{18} \mathrm{H}_{36} \mathrm{O}_{2}$ & 284 & 13.29 & Palmitic acid ester & $\begin{array}{l}\text { Antioxidant, Hemolytic, } \\
\text { Hypocholesterolemic, Flavor, Nematicide, } \\
\text { Antiandrogenic }\end{array}$ \\
\hline 21.53 & $\begin{array}{l}\text { Linolelaidic acid, methyl } \\
\text { ester }\end{array}$ & $\mathrm{C}_{19} \mathrm{H}_{34} \mathrm{O}_{2}$ & 294 & 2.41 & Fatty acid & No activity reported. \\
\hline 21.64 & 9,12,15-Octadecacatrienoic & $\mathrm{C}_{19} \mathrm{H}_{32} \mathrm{O}_{2}$ & 292 & 2.7 & Steroid & Antiarthritic, Anticancer, Hepatoprotective, \\
\hline
\end{tabular}




\begin{tabular}{|c|c|c|c|c|c|c|}
\hline 23.92 & $\begin{array}{l}\text { acid, methyl ester, (Z,Z,Z) } \\
\text { 12,15-Octadecadiynoic } \\
\text { acid, methyl ester }\end{array}$ & $\mathrm{C}_{19} \mathrm{H}_{30} \mathrm{O}_{2}$ & 290 & 0.50 & $\begin{array}{l}\text { Unsaturated fatty } \\
\text { acid ester }\end{array}$ & $\begin{array}{l}\text { Antimicrobial, Antiasthma, Diuretic. } \\
\text { No Activity reported. }\end{array}$ \\
\hline 24.64 & $\begin{array}{l}\text { Hexadecanoic acid, 2- } \\
\text { hydroxy-1- } \\
\text { (hydroxymethyl) ethyl } \\
\text { ester }\end{array}$ & $\mathrm{C}_{19} \mathrm{H}_{38} \mathrm{O}_{4}$ & 330 & 0.96 & Amino compound & Antimicrobial \\
\hline 24.90 & Diisooctyl phthalate & $\begin{array}{l}\mathrm{C}_{8} \mathrm{H}_{4}\left(\mathrm{C}_{8} \mathrm{H}_{1}\right. \\
\left.{ }_{7} \mathrm{COO}\right)_{2}\end{array}$ & 390 & 53.84 & $\begin{array}{l}\text { Plasticizer } \\
\text { compound }\end{array}$ & Antimicrobial, Antifouling \\
\hline 25.07 & $\begin{array}{l}\text { Docosanoic acid, ethyl } \\
\text { ester }\end{array}$ & $\mathrm{C}_{24} \mathrm{H}_{48} \mathrm{O}_{2}$ & 368 & 0.69 & Fatty ester & No Activity reported. \\
\hline 28.59 & Stigmasterol & $\mathrm{C}_{2}{ }_{9} \mathrm{H}_{48} \mathrm{O}$ & 412 & 2.57 & Steroid & $\begin{array}{l}\text { Antioxidant, hypoglycemic and thyroid } \\
\text { inhibiting properties, precursor of } \\
\text { progesterone, antimicrobial, anticancer, } \\
\text { antiarthritic, antiasthama, anti- } \\
\text { inflammatory, diuretic }\end{array}$ \\
\hline
\end{tabular}

Activity Source: -Dr. Duke's Phytochemical and Ethnobotanical Databases
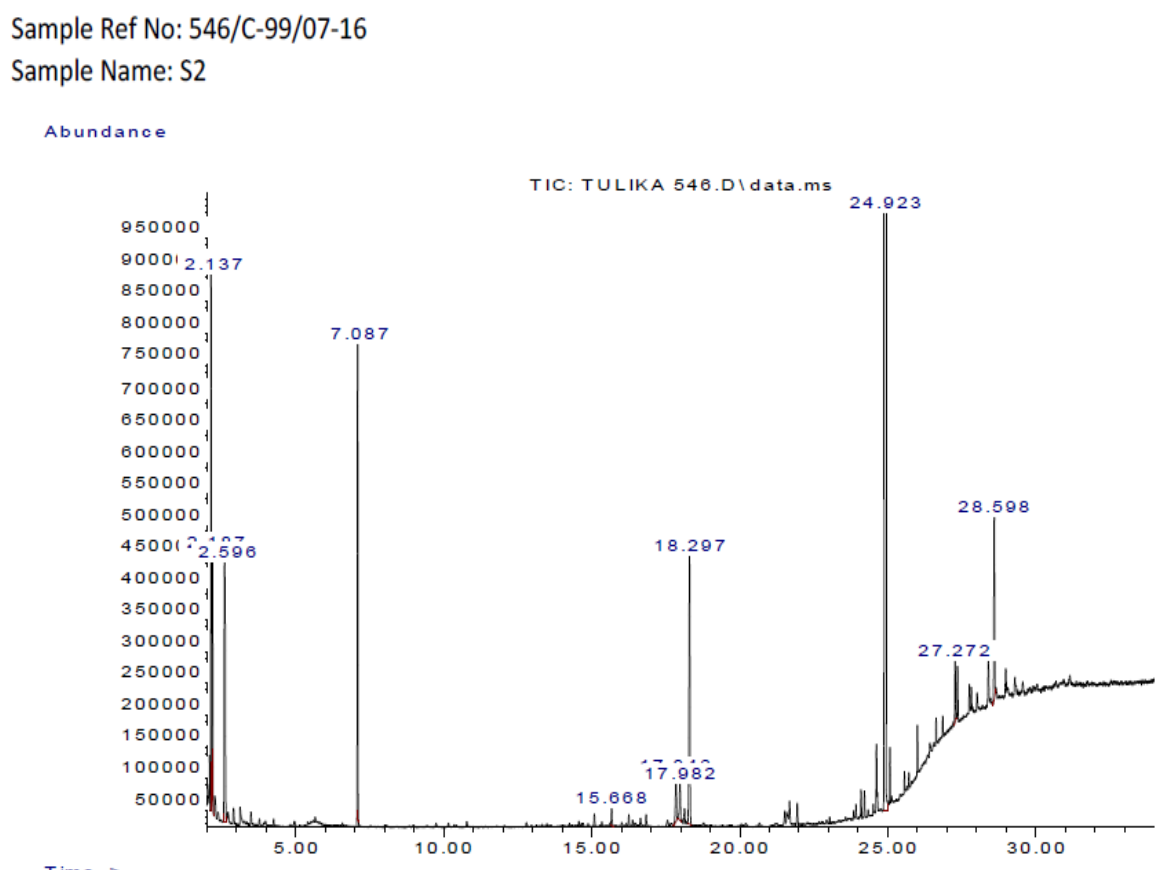

Fig. 2: GC-MS spectra of ethanolic extract of Root $P$. stratiotes

Table 2: Compound present in the ethanolic extract of the root of Pistia stratiotes

\begin{tabular}{|c|c|c|c|c|c|c|}
\hline RT & Name of compound & MF & MW & $\begin{array}{l}\text { Peak } \\
\text { area } \%\end{array}$ & $\begin{array}{l}\text { Compound } \\
\text { nature }\end{array}$ & Activity \\
\hline 2.13 & Isobutyl alcohol & $\mathrm{C}_{4} \mathrm{H}_{10} \mathrm{O}$ & 74 & 1.70 & Alcohol & Biofuel \\
\hline 2.59 & Ethane, 1,1-diethoxy & $\mathrm{C}_{6} \mathrm{C}_{14} \mathrm{O}_{2}$ & 118 & 1.79 & Ether & Flavoring agent \\
\hline 7.08 & $\begin{array}{l}\text { Propane,1,1-diethoxy-2- } \\
\text { methyl }\end{array}$ & $\mathrm{C}_{8} \mathrm{H}_{18} \mathrm{O}_{2}$ & 146 & 1.61 & Ether & No activity reported. \\
\hline 15.66 & $\begin{array}{l}\text { Octadecanoic acid, ethyl } \\
\text { ester }\end{array}$ & $\mathrm{C}_{20} \mathrm{H}_{40} \mathrm{O}_{2}$ & 312 & 0.07 & Fatty ester & No activity reported. \\
\hline 17.84 & n-Hexadecanoic acid & $\mathrm{C}_{16} \mathrm{H}_{32} \mathrm{O}_{2}$ & 256 & 0.30 & $\begin{array}{l}\text { Palmitric acid } \\
\text { (saturated } \\
\text { fatty acid) }\end{array}$ & $\begin{array}{l}\text { Antioxidant, antipsychotic, Hypocholesterolemic, } \\
\text { Nematicide, Pesticide, Lubricant, Antiandrogenic, } \\
\text { Hemolytic, 5-alpha reductase Inhibitor. }\end{array}$ \\
\hline 17.98 & $\begin{array}{l}\text { E-11-Hexadecanoic acid, } \\
\text { ethyl ester }\end{array}$ & $\mathrm{C}_{18} \mathrm{H}_{34} \mathrm{O}_{2}$ & 282 & 0.24 & Stearic acid & Antifungal, Antitumour, Antibacterial \\
\hline 18.29 & $\begin{array}{l}\text { Hexadecanoic acid, ethyl } \\
\text { ester }\end{array}$ & $\mathrm{C}_{18} \mathrm{H}_{36} \mathrm{O}_{2}$ & 284 & 1.63 & $\begin{array}{l}\text { Palmitic acid } \\
\text { ester }\end{array}$ & $\begin{array}{l}\text { Antioxidant, Hemolytic, Hypocholesterolemic, } \\
\text { Flavor, Nematicide, Antiandrogenic }\end{array}$ \\
\hline 24.92 & Bis (2-ethylhexyl) phthalate & $\mathrm{C}_{8} \mathrm{H}_{4}\left(\mathrm{C}_{8} \mathrm{H}_{17} \mathrm{COO}\right)_{2}$ & 390 & 91.49 & $\begin{array}{l}\text { Plasticizer } \\
\text { compound }\end{array}$ & Solvent in glowstick. \\
\hline 27.27 & $\begin{array}{l}\text { 1-Monolinoleoylglycerol } \\
\text { trimethylsilyl ether }\end{array}$ & $\mathrm{C}_{27} \mathrm{H}_{54} \mathrm{O}_{4} \mathrm{Si}_{2}$ & 498 & 0.22 & Steroid & $\begin{array}{l}\text { Antiarthritic, Anticancer Hepatoprotective, } \\
\text { Antimicrobial, } \\
\text { Antiasthma, Diuretic }\end{array}$ \\
\hline 28.59 & Ethyl iso-allocholate & $\mathrm{C}_{26} \mathrm{H}_{44} \mathrm{O}_{5}$ & 436 & 0.92 & Steroid & $\begin{array}{l}\text { Antimicrobial, Diuretic, Anti-inflammatory, } \\
\text { Antiasthma }\end{array}$ \\
\hline
\end{tabular}

Activity Source: -Dr. Duke's Phytochemical and Ethnobotanical Databases 

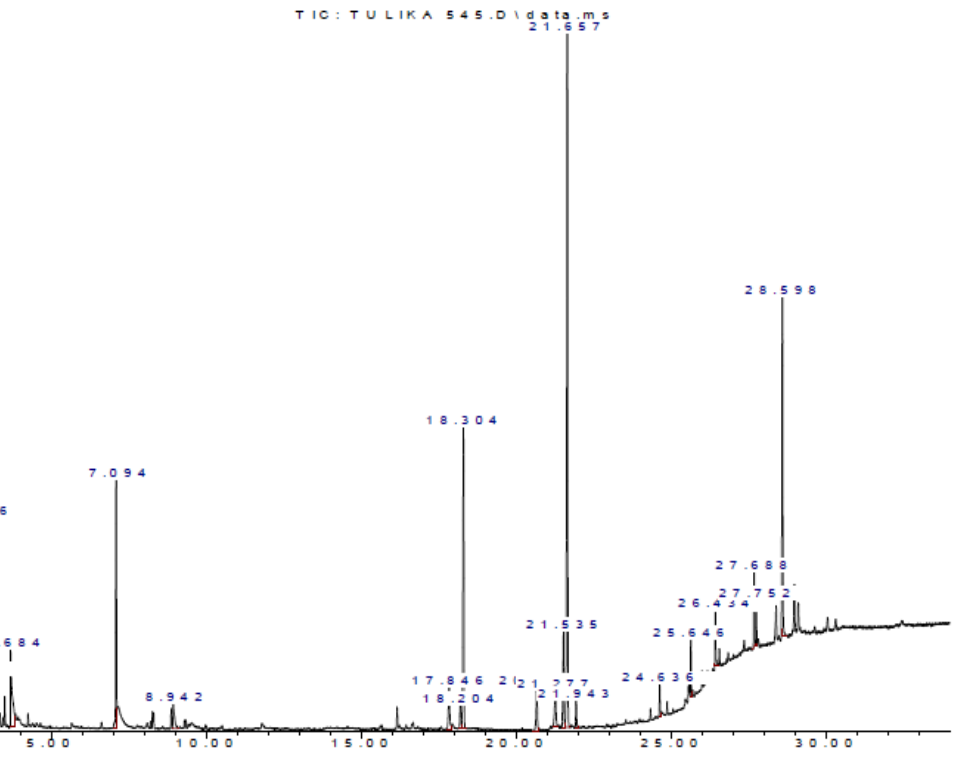

Fig. 3: GC-MS spectra of ethanolic extract of Leaf E. crassipes

Table 3: Compound present in the ethanolic extract of Leaf of Eichhornia crassipes

\begin{tabular}{|c|c|c|c|c|c|c|}
\hline RT & Name of compound & MF & MW & $\begin{array}{l}\text { Peak } \\
\text { area \% }\end{array}$ & $\begin{array}{l}\text { Compound } \\
\text { nature }\end{array}$ & Activity \\
\hline 2.59 & Ethane, 1,1-diethoxy & $\mathrm{C}_{6} \mathrm{H}_{14} \mathrm{O}_{2}$ & 118 & 9.77 & Ether & Flavoring agent \\
\hline 7.09 & $\begin{array}{l}\text { Propane,1,1-diethoxy-2- } \\
\text { methyl }\end{array}$ & $\mathrm{C}_{8} \mathrm{H}_{18} \mathrm{O}_{2}$ & 146 & 7.10 & Ether & No activity reported. \\
\hline 17.84 & n-Hexadecanoic acid & $\mathrm{C}_{16} \mathrm{H}_{32} \mathrm{O}_{2}$ & 256 & 2.34 & $\begin{array}{l}\text { Palmitric acid } \\
\text { (saturated } \\
\text { fatty acid) }\end{array}$ & $\begin{array}{l}\text { Antioxidant, Hypocholesterolemic, Nematicide, } \\
\text { Pesticide, Lubricant, Antiandrogenic, Hemolytic, 5- } \\
\text { alpha reductase inhibitor, antipsychotic }\end{array}$ \\
\hline 18.20 & $\begin{array}{l}\text { E-11-Hexadecanoic acid, } \\
\text { ethyl ester }\end{array}$ & $\mathrm{C}_{18} \mathrm{H}_{34} \mathrm{O}_{2}$ & 282 & 1.04 & Stearic acid & Antifungal, Antitumour, Antibacterial \\
\hline 18.30 & Palmitic acid, ethyl ester & $\mathrm{C}_{18} \mathrm{H}_{36} \mathrm{O}_{2}$ & 284 & 12.09 & Stearic acid & Antifungal, Antitumour, Antibacterial \\
\hline 20.66 & Phytol & $\mathrm{C}_{20} \mathrm{H}_{44} \mathrm{O}$ & 296 & 2.12 & Diterpene & $\begin{array}{l}\text { Antimicrobial, Anti-inflammatory, Anticancer, } \\
\text { Diuretic, Antifungal against } S \text {. typhi, resistant } \\
\text { gonorrhea, joint dislocation, headache, hernia, } \\
\text { stimulant and antimalarial }\end{array}$ \\
\hline 21.27 & $9,12,15$-Octadecatrienal & $\mathrm{C}_{18} \mathrm{H}_{30} \mathrm{O}$ & 262 & 2.10 & Steroid & $\begin{array}{l}\text { Antiarthritic, Anticancer, Hepatoprotective, } \\
\text { Antimicrobial, Antiasthma, Diuretic }\end{array}$ \\
\hline 21.53 & $\begin{array}{l}\text { 9,12-Octadecadienoic acid, } \\
\text { ethyl ester }\end{array}$ & $\mathrm{C}_{20} \mathrm{H}_{36} \mathrm{O}_{2}$ & 308 & 3.79 & $\begin{array}{l}\text { Polyenoic fatty } \\
\text { acid }\end{array}$ & $\begin{array}{l}\text { Hepatoprotective, antihistaminic, } \\
\text { hypocholesterolemic, antieczemic }\end{array}$ \\
\hline 21.65 & Linolenic acid, ethyl ester & $\mathrm{C}_{20} \mathrm{H}_{34} \mathrm{O}_{2}$ & 306 & 26.26 & $\begin{array}{l}\text { Linoleic acid } \\
\text { ethyl ester }\end{array}$ & $\begin{array}{l}\text { Hypocholesterolemic, Nematicide, Antiarthritic, } \\
\text { Hepatoprotective Antiandrogenic, } \\
\text { Hypocholesterolemic, 5-Alpha reductaseinhibitor, } \\
\text { Antihistaminic, Anticoronary, Insectifuge, } \\
\text { Antieczemic, Antiacne }\end{array}$ \\
\hline 21.94 & Stearic acid, ethyl ester & $\mathrm{C}_{20} \mathrm{H}_{40} \mathrm{O}_{2}$ & 312 & 0.98 & Fatty ester & No activity reported. \\
\hline 24.63 & $\begin{array}{l}\text { Hexadecanoic acid, 2- } \\
\text { hydroxy-1- } \\
\text { (hydroxymethyl) ethyl } \\
\text { ester }\end{array}$ & $\mathrm{C}_{19} \mathrm{H}_{38} \mathrm{O}_{4}$ & 330 & 0.87 & $\begin{array}{l}\text { Amino } \\
\text { compound }\end{array}$ & Antimicrobial \\
\hline 25.64 & $\alpha$-Glyceryl linolenate & $\mathrm{C}_{21} \mathrm{H}_{36} \mathrm{O}_{4}$ & 352 & 1.35 & $\begin{array}{l}\text { Fatty acid } \\
\text { Ester }\end{array}$ & Cosmetic, Colouring agent. \\
\hline 26.43 & $\begin{array}{l}\text { 1-Monolinoleoylglycerol } \\
\text { trimethylsilyl ether }\end{array}$ & $\mathrm{C}_{27} \mathrm{H}_{54} \mathrm{O}_{4} \mathrm{Si}_{2}$ & 498 & 1.52 & Steroid & $\begin{array}{l}\text { Antiarthritic, Anticancer, Hepatoprotective, } \\
\text { Antimicrobial, Antiasthma, Diuretic. }\end{array}$ \\
\hline 27.68 & $\begin{array}{l}\text { Linoleic acid, 2,3-bis-(0- } \\
\text { TMS)-propyl ester }\end{array}$ & $\mathrm{C}_{27} \mathrm{H}_{54} \mathrm{O}_{4} \mathrm{Si}_{2}$ & 498 & 1.98 & Steroid & $\begin{array}{l}\text { Antiarthritic, Anticancer, Hepatoprotective, } \\
\text { Antimicrobial, Antiasthma, Diuretic }\end{array}$ \\
\hline 28.59 & Stigmasterol & $\mathrm{C}_{29} \mathrm{H}_{48} \mathrm{O}$ & 412 & 11.39 & Steroid & $\begin{array}{l}\text { Antioxidant, hypoglycemic and thyroid inhibiting } \\
\text { properties, precursor of progesterone, antimicrobial, } \\
\text { anticancer, antiarthritic, antiasthama, anti } \\
\text { inflammatory, diuretic }\end{array}$ \\
\hline
\end{tabular}

Activity Source: -Dr. Duke's Phytochemical and Ethnobotanical Database 


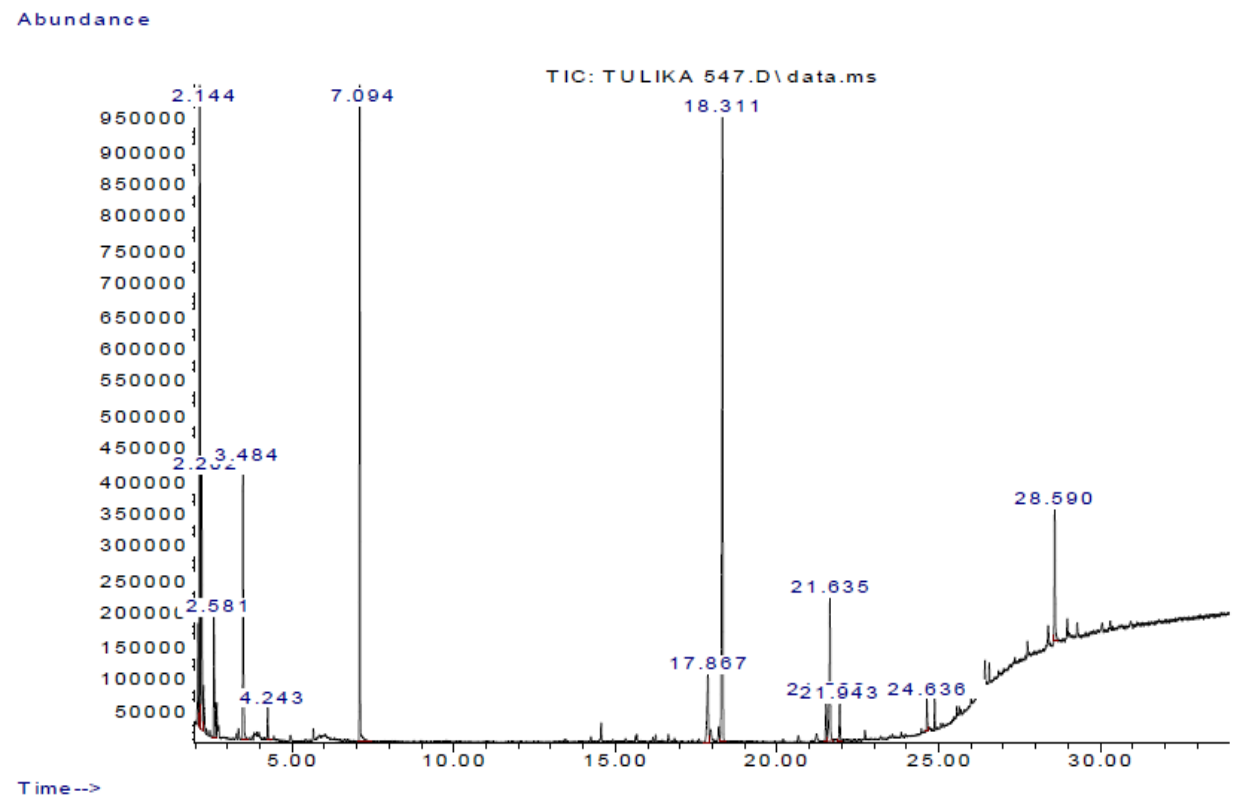

Fig. 4: GC-MS spectra of ethanolic extract of petiole of E. crassipes

Table 4: Compound present in the ethanolic extract of the petiole of Eichhornia crassipes

\begin{tabular}{|c|c|c|c|c|c|c|}
\hline RT & Name of compound & $\begin{array}{l}\text { Molecular } \\
\text { formula }\end{array}$ & $\begin{array}{l}\text { Molecular } \\
\text { weight }\end{array}$ & $\begin{array}{l}\text { Peak } \\
\text { area \% }\end{array}$ & $\begin{array}{l}\text { Compound } \\
\text { nature }\end{array}$ & Activity \\
\hline 2.14 & Isobutyl alcohol & $\mathrm{C}_{4} \mathrm{H}_{10} \mathrm{O}$ & 74 & 28.82 & Alcohol & Biofuel \\
\hline 2.58 & Ethane, 1,1-diethoxy & $\mathrm{C}_{6} \mathrm{H}_{14} \mathrm{O}_{2}$ & 118 & 4.15 & Ether & Flavoring agent \\
\hline 3.48 & $\begin{array}{l}\text { Formic acid,1-methylethyl } \\
\text { ester }\end{array}$ & $\mathrm{C}_{4} \mathrm{H}_{8} \mathrm{O}_{2}$ & 88 & 7.6 & carboxylic acid & $\begin{array}{l}\text { Preservative, an antibacterial agent, treatment } \\
\text { for warts. }\end{array}$ \\
\hline 4.24 & Ethonal, 2-propoxy & $\mathrm{C}_{5} \mathrm{H}_{12} \mathrm{O}_{2}$ & 104 & 0.97 & Ether & Solvent, Paints and Coating. \\
\hline 7.09 & $\begin{array}{l}\text { Propane,1,1-diethoxy-2- } \\
\text { methyl }\end{array}$ & $\mathrm{C}_{8} \mathrm{C}_{18} \mathrm{O}_{2}$ & 146 & 16.84 & Ether & No activity reported. \\
\hline 17.86 & n-Hexadecanoic acid & $\mathrm{C}_{16} \mathrm{H}_{32} \mathrm{O}_{2}$ & 256 & 3.82 & $\begin{array}{l}\text { Palmitric acid } \\
\text { (saturated fatty } \\
\text { acid) }\end{array}$ & $\begin{array}{l}\text { Antioxidant, Pesticide, Hypocholesterolemic, } \\
\text { Nematicide, Lubricant, Antiandrogenic, } \\
\text { Hemolytic, 5-alpha reductase inhibitor, } \\
\text { antipsychotic }\end{array}$ \\
\hline 18.31 & $\begin{array}{l}\text { Hexadecanoic acid, ethyl } \\
\text { ester }\end{array}$ & $\mathrm{C}_{18} \mathrm{H}_{36} \mathrm{O}_{2}$ & 284 & 23.7 & $\begin{array}{l}\text { Palmitic acid } \\
\text { ethyl ester }\end{array}$ & $\begin{array}{l}\text { Antioxidant, Nematicide, Hypocholesterolemic, } \\
\text { Pesticide, Antiandrogenic, flavor, Hemolytic, } \\
\text { Alpha reductase inhibitor }\end{array}$ \\
\hline 21.52 & $\begin{array}{l}\text { Linolelaidic acid, methyl } \\
\text { ester }\end{array}$ & $\mathrm{C}_{19} \mathrm{H}_{34} \mathrm{O}_{2}$ & 294 & 1.62 & Fatty acid & No activity reported. \\
\hline 21.63 & $\begin{array}{l}\text { 9,12,15-Octadecatrienoic } \\
\text { acid, ethyl ester,(Z,Z,Z)- }\end{array}$ & $\mathrm{C}_{20} \mathrm{H}_{34} \mathrm{O}_{2}$ & 306 & 5.50 & $\begin{array}{l}\text { Linolenic acid } \\
\text { ester } \\
\text { compound }\end{array}$ & $\begin{array}{l}\text { Anti-inflammatory, Cancer preventive, } \\
\text { Hepatoprotective }\end{array}$ \\
\hline 21.94 & $\begin{array}{l}\text { Octadecanoic acid, ethyl } \\
\text { ester }\end{array}$ & $\mathrm{C}_{20} \mathrm{H}_{40} \mathrm{O}_{2}$ & 312 & 1.32 & Fatty ester & No activity reported. \\
\hline 24.63 & $\begin{array}{l}\text { Hexadecanoic acid, 2- } \\
\text { hydroxy-1- } \\
\text { (hydroxymethyl) ethyl } \\
\text { ester }\end{array}$ & $\mathrm{C}_{19} \mathrm{H}_{38} \mathrm{O}_{4}$ & 330 & 0.94 & $\begin{array}{l}\text { Amino } \\
\text { compound }\end{array}$ & Antimicrobial \\
\hline 28.59 & Ethyl iso-allocholate & $\mathrm{C}_{26} \mathrm{H}_{44} \mathrm{O}_{5}$ & 436 & 4.65 & Steroid & $\begin{array}{l}\text { Antimicrobial, Diuretic, Anti-inflammatory, } \\
\text { Antiasthma }\end{array}$ \\
\hline
\end{tabular}

Activity Source: -Dr. Duke's Phytochemical and Ethnobotanical Databases

\section{DISCUSSION}

The ethanol extract contains various useful compounds having pharmacological activity. Phytol is a diterpene compound and it may be act as an antimicrobial, anti-inflammatory, anti-cancer and diuretic. Phytol gives good preventive and therapeutic results against arthritis and shows that reactive oxygen species constitute a promising novel class of pharmaceuticals for the treatment of rheumatoid arthritis and other chronic inflammatory diseases [6]. Stigmasterol is an unsaturated plant sterol and act as a precursor in the manufacture of semi-synthetic progesterone, a valuable human hormone that plays an important physiological role in the regulatory and tissue rebuilding mechanisms related to oestrogen effects, as well as acting as an intermediate in the biosynthesis of androgens, oestrogens and corticoids. It is also used as the precursor of Vitamin $\mathrm{D}_{3}[7]$. 
Sample Ref No: 549/C-102/07-16

Sample Name: S5

Abundance

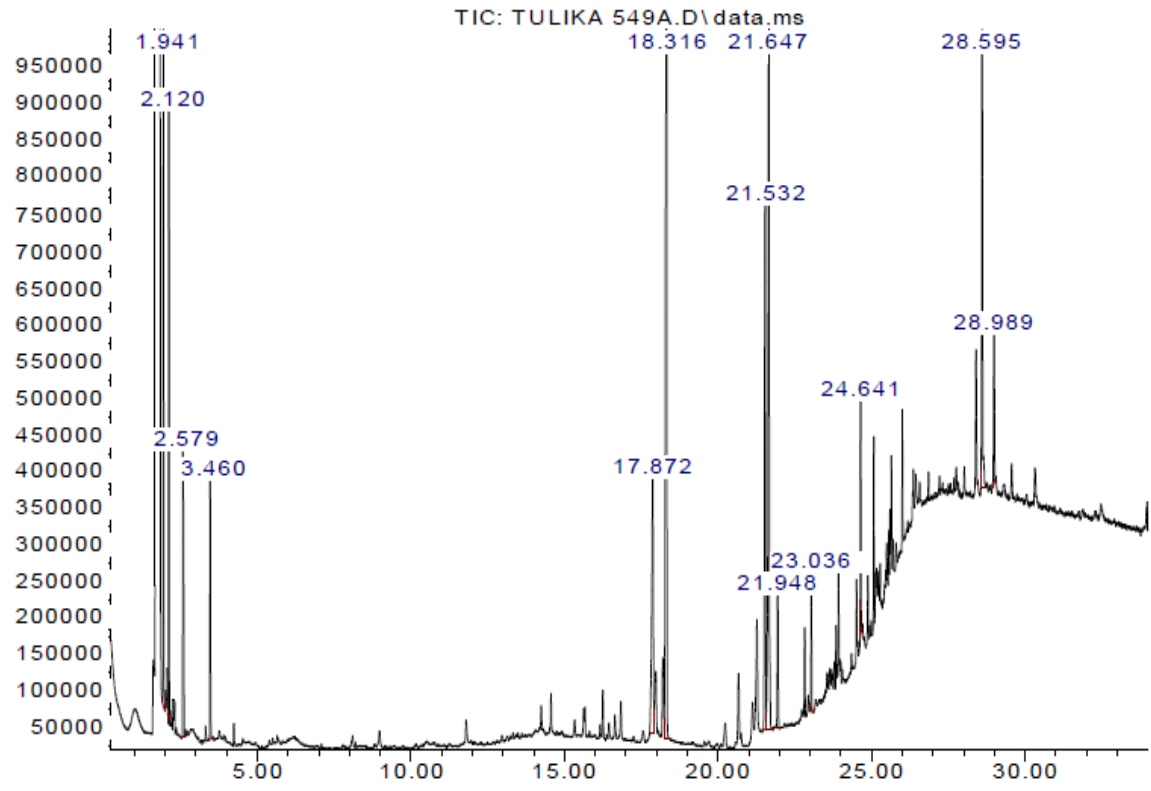

Time-->

Fig. 5: GC-MS Spectra of ethanolic extract of Root of $E$. crassipes

Table 5: Compound present in the ethanolic extract of the Root of Eichhornia crassipes

\begin{tabular}{|c|c|c|c|c|c|c|}
\hline RT & Name of compound & MF & MW & $\begin{array}{l}\text { Peak } \\
\text { area } \%\end{array}$ & $\begin{array}{l}\text { Compound } \\
\text { nature }\end{array}$ & Activity \\
\hline 1.94 & Propyl alcohol & $\mathrm{C}_{3} \mathrm{H}_{8} \mathrm{O}$ & 60 & 5.53 & Alcohol & Engine fuel \\
\hline 2.12 & 1-Propanol, 2-methyl & $\mathrm{C}_{4} \mathrm{H}_{10} \mathrm{O}$ & 74 & 4.85 & Alchol & Industrial Solvent \\
\hline 2.57 & Ethane, 1,1-diethoxy & $\mathrm{C}_{6} \mathrm{H}_{14} \mathrm{O}_{2}$ & 118 & 5.14 & Ether & Flavoring agent \\
\hline 3.46 & $\begin{array}{l}\text { Formic acid,1-methyl ethyl } \\
\text { ester }\end{array}$ & $\mathrm{C}_{4} \mathrm{H}_{8} \mathrm{O}_{2}$ & 88 & 2.95 & carboxylic acid & $\begin{array}{l}\text { Preservative, an antibacterial agent, treatment for } \\
\text { warts. }\end{array}$ \\
\hline 17.87 & n-Hexadecanoic acid & $\mathrm{C}_{16} \mathrm{H}_{32} \mathrm{O}_{2}$ & 256 & 7.98 & $\begin{array}{l}\text { Palmitric acid } \\
\text { (saturated fatty } \\
\text { acid) }\end{array}$ & $\begin{array}{l}\text { Antioxidant, Hypocholesterolemic, Nematicide, } \\
\text { Pesticide, Lubricant, Antiandrogenic, Hemolytic, } \\
\text { 5-alpha reductase inhibitor, antipsychotic }\end{array}$ \\
\hline 18.31 & $\begin{array}{l}\text { Hexadecanoic acid, ethyl } \\
\text { ester }\end{array}$ & $\mathrm{C}_{18} \mathrm{H}_{36} \mathrm{O}_{2}$ & 284 & 28.33 & $\begin{array}{l}\text { Palmitic acid } \\
\text { ester }\end{array}$ & $\begin{array}{l}\text { Antioxidant, Hemolytic Hypocholesterolemic, } \\
\text { Flavor, Nematicide, Antiandrogenic, }\end{array}$ \\
\hline 21.53 & $\begin{array}{l}\text { 9,12-Octadecadienoic acid, } \\
\text { ethyl ester }\end{array}$ & $\mathrm{C}_{20} \mathrm{H}_{36} \mathrm{O}_{2}$ & 308 & 9.99 & $\begin{array}{l}\text { Unsaturated fatty } \\
\text { acid ester }\end{array}$ & Anti-inflammatory,Cancer preventive \\
\hline 21.64 & Linolenic acid, ethyl ester & $\mathrm{C}_{20} \mathrm{H}_{34} \mathrm{O}_{2}$ & 306 & 17.65 & $\begin{array}{l}\text { Unsaturated fatty } \\
\text { acid ester }\end{array}$ & Anti-inflammatory,Cancer preventive \\
\hline 21.94 & $\begin{array}{l}\text { Octadecanoic acid, ethyl } \\
\text { ester }\end{array}$ & $\mathrm{C}_{20} \mathrm{H}_{40} \mathrm{O}_{2}$ & 312 & 2.27 & Fatty ester & No Activity reported. \\
\hline 23.03 & Octadecane, 6-methyl & $\mathrm{C}_{19} \mathrm{H}_{40}$ & 268 & 1.66 & Alkane & Absorbant \\
\hline 24.64 & $\begin{array}{l}\text { Hexadecanoic acid, } 2 \text { - } \\
\text { hydroxy-1-(hydroxymethyl) } \\
\text { ethyl ester }\end{array}$ & $\mathrm{C}_{19} \mathrm{H}_{38} \mathrm{O}_{4}$ & 330 & 2.51 & Amino compound & Antimicrobial \\
\hline 28.59 & $\begin{array}{l}\text { Cholesta-22,24-diene-5-ol, } \\
\text { 4,4-dimethyl }\end{array}$ & $\mathrm{C}_{29} \mathrm{H}_{48} \mathrm{O}$ & 412 & 8.39 & Steroid & Antibacterial, trypanocidal activity \\
\hline 28.98 & $\begin{array}{l}\text { 1-Monolinoleoylglycerol } \\
\text { trimethylsilyl ether }\end{array}$ & $\mathrm{C}_{27} \mathrm{H}_{54} \mathrm{O}_{4} \mathrm{Si}_{2}$ & 498 & 2.71 & Steroid & $\begin{array}{l}\text { Antiarthritic, Anticancer, Hepatoprotective, } \\
\text { Antimicrobial, Antiasthma, Diuretic }\end{array}$ \\
\hline
\end{tabular}

Activity Source: -Dr. Duke's Phytochemical and Ethnobotanical Databases

1-Monolinoleoylglycerol trimethylsilyl ether is the common compound in the roots of both the plant show many biological activities such as Antiarthritic, Anticancer, Hepatoprotective, Antimicrobial, Antiasthma, Diuretic, antioxidant, anti-inflammatory and anti-diabetic [8]. 9, 12-Octadecadienoic acid, ethyl ester, is a polyenoic fatty acid compound and it acts as an antihistaminic, hepatoprotective, hypocholesterolemic and antieczemic [9]. 9,12,15-
Octadecatrienoic acid, methyl ester, (Z,Z,Z)-is a polyenoic fatty acid compound and it may be acts as an anti-inflammatory, hypocholesterolemic, cancer preventive, hepatoprotective, nematicide, insectifuge, anti-histaminic, anti arthritic, anti-coronary, anti eczemic, anti-acne, 5-alpha reductase inhibitor and antiandrogenic [10]. Diisooctyl phthalate is a plasticizer compound; it may be acts as an antimicrobial and antifouling [11]. Ethyl iso- 
allocholate is a sterol compound and it uses as an antibacterial, antioxidant, antitumor, cancer preventive, pesticide and chemopreventive agent. Cholesta-22, 24-dien-5-ol, 4,4-dimethyl-is a steroid compound possess Antimicrobial, anti-inflammatory, anticancer, diuretic, antiarthritic, antiasthma and trypanocidal activity. Hexadecanoic acid, 2-hydroxy-1-(hydroxymethyl) ethyl ester found in the leaves of both plant extract act as Hemolytic, pesticide, flavour, antioxidant [12].

\section{CONCLUSION}

GC-MS analysis of ethanol extract of leaf of $P$. stratiotes and E. crassipes revealed the presence of secondary metabolites of anticancerous, antimicrobial, antioxidant, antidandruff, antiproliferative activities and provides a potential source of the industrial application. We concluded that the biological values of $P$. stratiotes and E. crassipes contain pharmacologically active compounds that may enhance its use as a traditional drug.

\section{ACKNOWLEDGEMENT}

The authors are grateful to Kishor Potekar and K. P Jain, Padmaja Aerobiologicals (P) Ltd. Plot no. 36, Sector-24, Near Bank of India, Turbhe, Navi Mumbai 400705. Maharashtra. India for providing the laboratory facilities (GC-MS) and support to carry out the work.

\section{CONFLICT OF INTERESTS}

Declare none

\section{REFERENCES}

1. Bigoniya P, Singh CS, Shukla A. Pharmacognostical and physicochemical standardization of ethnopharmacologically important seeds of Lepidium sativum Linn. and Wrightia tinctoria R. Br. Indian J Nat Prod Resour 2011;2 Suppl 4:46471.

2. Kirtikar KR, Basu BD. Indian Medicinal Plants. Delhi: Sri Satguru Publications; 2000.
3. Kirtikar KK, BD Basu. The Indian medicinal plants. Dehradun: Oriental Enterprises; 2001. p. 3576-9.

4. Jayanthi P, Lalitha P. Reducing the power of the solvent extracts of Eichhornia crassipes (Mart.) Solms. Int J Pharm Pharm Sci 2011;3 Suppl 3:126-8.

5. Nair V, Kanfer I, Hoogmartens J. Determination of $\beta$-sitosterol and stigmasterol in oral dosage forms using high-performance liquid chromatography with evaporative light scattering detection. J Pharm Biomed Anal 2006;41 Suppl 3:731-7.

6. Ogunlesi M, Okiei W, Ofor E, Osibote AE. Analysis of the essential oil from the dried leaves of Euphorbia hirta Linn (Euphorbiaceae), a potential medication for asthma. Afr J Biotech 2009;8:7042-50.

7. Kametani T, Furuyama H. Synthesis of Vitamin D3 and related compounds Med Res Rev 1987;7 Suppl 2:147-71.

8. Senthil J, Rameashkannan MV, Mani P. Phytochemical profiling of ethonolic leaves extract of ipomoea sepiaria (Koenig Ex. Roxb). Int J Innovative Res Sci Eng Technol 2016;5 Suppl 3:3140-7.

9. Wu L, Gao H, Wang X, Ye J, Lu J. Analysis of the chemical composition of Chrysanthemum indicum flowers by GC/MS and HPTLC. J Med Plants Res 2010;4 Suppl 5:421-6.

10. Vohra A, Kaur H. Chemical investigation of medicinal plant Ajuga bracteosa. J Nat Prod Plant Resour 2011;1 Suppl 1:37-45.

11. Sangeetha J, Vijayalakshmi K. Determination of bioactive components of ethyl acetate fraction of Punica granatum Rind extract. Int J Pharm Sci Drug Res 2011;3 Suppl 2:116-22.

12. Duke's Phytochemical and Ethnobotanical Databases (19921996). U. S. Department of Agriculture, Agricultural Research Service. Available from: http://phytochem.nal.usda.gov. [Last accessed on 20 Mar 2017]

\section{How to cite this article}

- Tulika Tyagi, Mala Agarwal. GC-MS analysis of invasive aquatic weed, Pistia stratiotes L. and Eichhornia crassipes (mart.) solms. Int J Curr Pharm Res 2017;9(3):111-117. 